\title{
Moving to Cloud :A Cloud Transformation Guide for Wealth Management Firms
}

\author{
Nachiket Vaidya \\ IEEE,Principle Solutions Architect
}

\begin{abstract}
Cloud transformation has reached most sectors of industry and financial institutions have started to embrace this modern day technology trend and is seen as a critical tool for financial institutions to stay competitive in today's challenging business environment. Success with process re-engineering and efforts at digitalization with emerging technologies such as artificial intelligence are dependent on cloud computing.This paper highlights the opportunities for Cloud transformation within wealth management firms, aims to address the concerns and provides guiding principles to implement a cloud transformation strategy with success.
\end{abstract}

Keywords: Cloud Strategy, Cloud Transformation, Finance, Wealth Management, Digital Journeys

\section{INTRODUCTION}

Over the years Wealth Management offerings have been one of the most lucrative within the financial sector; primarily due to greater growth potential but also due to low capital requirements. The higher return on equity compared to any other retail offerings have always attracted the traditional customer with higher retention chances.However in a technology disruptive age, Wealth Management firms worldwide today face stiffer challenges than before; with ever-changing buying habits of the millennials and a challenging environment for investors and their advisors to find the right risk/return combinations. A new generation of investors are tasked to think differently about advice and bring new expectations from Wealth Management offerings, influencing the traditional patterns to purchase and consume wealth services. To accomplish this, many firms are exploring the potential of Cloud as a business asset to help transform the organization and revitalize the operating models,product/service offerings with focus on enhancing the customer experience and maximize growth. Cloud thus has become a key element for the digital change within the Wealth Management Sector.

\section{TRANSFORMATION CHALLENGES WITHIN THE WEALTH MANAGEMENT SECTOR}

Digital transformation is not a one-size-fits-all solution for wealth management firms since each firm has a unique value proposition. A firm's size and complexity present different challenges to transformation, leading to confusion as to where to start. Firms understand that digital transformation is critical to their evolution; however, they may face any number of transformation challenges, For example Firms engaging in experimental cloud projects may find it difficult to realize desired business outcomes. Even though the firms are open to the idea of embracing Cloud for digital transformation an overall commitment is needed to realize the full potential of cloud. A well formulated digital strategy with new gen technologies such as cloud can help overcome lot of transformational challenges like below-

\section{- Lack of Unified Transformation Strategy}

Organizational cultural reservations against embracing latest technology trends often lead to wealth management firms adopting horses for courses approaches rather than identifying a well formulated digital strategy for addressing targeted business models.

- Challenging Customers and Business Environment

Continuously changing buying habits of millennials, need for enhanced customer user experience and artificial intelligence versus human based advice models results in posing customer acquisition and retention challenges for the firms

\section{- Changing Regulations}

Newer and emerging regulations challenge the business models of the firms to change and adapt to newer expectations and can be restrained with limited digitization and legacy systems/applications. A flexible digital transformation model can help resolve this challenge.

\section{- Legacy Systems}

Legacy Applications have been long standing and perhaps the only source of any digitization within wealth management firms Integrating with legacy system/applications can often lead to increase implementation costs, and questions surrounding the value of the transformation for the firm.

\section{- Non Standard Data Storage}

Lack of Data hubs or decentralized Data Storage solutions lead to storage of different data with different data structures, duplication of data, and the use of unstructured and non-standard data often resulting in increased costs for businesses.

\section{- Manual Processes}

Lack of Automation and with virtualization not delivering expected results, firms often adopt 
manual processes with adhoc workarounds, this can become a hurdle while delivering high value customer experience and needs to be addressed through digital transformation processes.

\section{CLOUD OPPORTUNITIES}

Cloud within Wealth Management creates a lucrative proposition and can become a key element to overcome some of the long standing processes/concerns by reducing the over reliance on legacy systems. It can also help accelerate a company's digital transformation journey.Cloud offers several opportunities within Wealth Management domain like below-

- Agility to support for challenging business environments

- Flexibility to support on demand consumption thereby offering a greater cost control over the Operational Expenses(OPEX)

- Cost Reduction and improved cost predictions

- Centralized Data Storage creating a uniform standardized data structures across the organization

- Unified Document Storage Solution with diversified access model

- Enhanced Automation and virtualization thereby greatly reducing the reliance on manual processes

\section{BARRIERS FOR CLOUD IMPLEMENTATION}

Although cloud offers a lucrative proposition for driving a transformation journey, most firms explore the possibilities of using cloud only on an experimental basis and are reluctant to undertake full scale transformation due to several perceived challenges like -

\section{- Security}

Loss or leakage of information, unauthorized access, data encryption, breaches due to third party integrations and outdated software, data restorations and disaster recovery are few of the concerns when it comes to accepting Cloud transformation as a full scale strategy especially within Wealth Management Firms.

\section{How Cloud Transformation Can Help}

Cloud vendors have come a long way in addressing the security concerns especially when it comes to the financial sector. There are several security and access control mechanisms that allow data and user level segmentation restricting the users to allowed data and operations. Additionally, security models within the cloud are flexible enough to be enhanced and integrated with third party security vendors.

\section{- Regulations}

National regulatory authorities often mandate that data held by domestic companies should be kept only on servers in that country, and that access to data should only be possible from within the country. Furthermore, they may impose legal obligations relating to investigations or data recovery. This means that a cloud service provider has to use local servers, which creates a major challenge for the operating model of cloud providers.

\section{How Cloud Transformation Can Help}

Major cloud service providers have tried to increase their global footprint by building more data centres in new locations close to their customers. This helps banks meet some of the regulatory requirements by having data physically located in the same country.

\section{- Costs Associated with Legacy App Modernization}

Most of the firms heavily rely heavily on legacy systems/applications for the core functionality and sections within the organization fear the costs of moving the legacy applications to cloud might be higher than the actual business benefits.

\section{How Cloud Transformation Can Help}

Most cloud vendors offer pay as you go cost structures which allows a better cost visibility and predictions for overall costs associated with modernizing legacy applications.

\section{GUIDING PRINCIPLES FOR SUCCESS WITH CLOUD IMPLEMENTATION}

Cloud transformations exercise should not be treated as just a project. To benefit fully from the advantages of the cloud, organisations need a digital transformation that goes well beyond a one-off project. With a phased approach and below guiding principles firms are bound to achieve more success with their transformation journey.

\section{- Cloud strategy}

Create a target business model and define your objectives for the cloud. Articulate the benefits - better cost predictions, security benefits, better risk management, ROIs. and lower capital investment. Clearly articulate the KPI to get organizational wide buy in. Cloud strategy should always align with business goals of the firms with increased IT benefits and operational intelligence.

\section{- Risk Management}

Moving to the cloud transfers some of the responsibilities for risk management to the third party cloud service provider. However, it is only the management of the risks that is transferred while the accountability for the risks still resides with the firm, and not the cloud service provider. The firm's operational risk management 
framework must therefore take account of the special circumstances arising from cloud service adoption. An important element of the framework should be to classify the information assets - such as intellectual property, customer databases and financial information - so that the inherent risks can be managed. Auditing the cloud environment along with business continuity plan(BCP), exit strategy and IT service management procedures and controls, and a redesigned operating model to ensure the right team structure and capabilities should be identified and put in place to manage the cloud services.

\section{- Governance}

Governance processes relating to the use of cloud services should be developed: who is able to request them, how many resources can be provided, and what approval(s) are required. In addition to setting quotas, providing visibility and reporting usage will help to hold users accountable. Organisations should therefore establish a robust cloud governance structure, Establishing governance and controls provides direction for the adoption of the cloud by an organisation. Structured governance is essential to monitor performance continually, improve service effectiveness, and align investments with business objectives.

\section{- Cloud Migration}

Applications may follow different migration paths, ranging from a simple 'lift-and-shift' where applications from firms' data centers are re-hosted in a cloud environment without further amendments. Another approach is a complete refactoring of the applications using 'cloud native' components. Each approach has pros and cons: for instance a complete refactoring is costly and creates vendor lock-in, but it also allows applications to leverage fully the capabilities of the cloud such as elasticity, high availability and high resilience and governance. Organisations should perform detailed evaluation of cost and risk factors of each approach to conduct migration in an efficient manner to avoid parallel operations and cost overrun.

\section{CONCLUSION}

Cloud provides transformative opportunities for organisations and is a vital competitive component in today's challenging marketplace. Cloud is not an easy technology to adopt, but the potential benefits and opportunities outweigh the challenges and risks associated with cloud transformation. To maximise cloud's added value, an organisation should follow a structured approach, starting with the definition of a clear strategy (and involving a wide range of stakeholders), clarity of vision and expectations, knowledge of options, understanding of business drivers (both opportunities and risks), proper planning, disciplined execution and ongoing governance and management.

\section{REFERENCES}

[1] KPMG -How to manage five key cloud computingrisks https://assets.kpmg/content/dam/kpmg/ca/pdf/2018/03/cloudcomputing-risks-canada.pdf

[2] Deloitte, "Secure and Private Computing for Banks on a Cloud Platform,

[3] Google, "BNP Paribas Fortis: Aligning marketing teams and resources to improve productivity," Google, [Online]. Available: https://cloud.google.com/customers/ bnp-paribas-fortis/.

[4] CGI-Digital Transformation reaches Wealth Management https://www.cgi.com/sites/default/files/2019-02/digitaltransformation-reaches-wealth-management-en.pdf 